\title{
In die Gänge gekommen: Kooperation von Zivilgesellschaft und Stadtpolitik
}

\author{
Rezension zu Michael Ziehl (2020): Koproduktion urbaner Resilienz. Das Gängeviertel \\ in Hamburg als Reallabor für eine zukunftsfähige Stadtentwicklung mittels \\ Kooperation von Zivilgesellschaft, Politik und Verwaltung. Hamburg: jovis.
}

KOPRODUKTION URBANER RESILIENZ

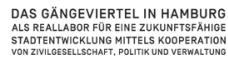

Lovis

Abb. 1 Titel des Buches (jovis Verlag)
Schlagworte wie Kooperation, Koproduktion und Kollaboration sind in Planungswissenschaften und Architektur schwer in Mode. Selten wird allerdings dargelegt, was diese Art(en) der Zusammenarbeit zwischen zivilgesellschaftlichen Akteuren auf der einen Seite und staatlichen Akteuren aus Politik und Verwaltung auf der anderen Seite von gewöhnlichen Formen der Partizipation und Bürger_innenbeteiligung unterscheidet. Anders im Buch von Michael Ziehl: Ihm gelingt es, anhand eines Fallbeispiels die intensive Zusammenarbeit zwischen Aktivist_innen rund ums Hamburger Gängeviertel und verschiedenen städtischen Institutionen detailliert nachzuzeichnen und damit den qualitativen Unterschied zwischen Kooperation und Beteiligung nachvollziehbar zu machen. Solche Kooperationen zur Entwicklung ausgewählter städtischer Räume oder zur Durch- und Umsetzung stadt- und wohnungspolitischer Maßnahmen sind (noch) recht selten. Ähnliche Beispiele für Kooperationen sind neben dem Gängeviertel die PlanBude in Hamburg, die Entwicklung des Dragonerareals in Berlin oder die Zusammenarbeit zwischen dem Netzwerk für gemeinschaftliches Wohnen e. V. und dem Amt für Wohnungswesen in Frankfurt am Main sowie das gemeinsam von der Stadt Leipzig und verschiedenen Initiativen zum Thema gemeinschaftliches Wohnen gegründete Netzwerk Leipziger Freiheit.

Solche Kooperationen können, so das Anliegen von Michael Ziehl, von den Erfahrungen im Gängeviertel und deren wissenschaftlicher Aufarbeitung lernen. Mit diesem Ansinnen hat der Autor seine Studie als transdisziplinäres Projekt angelegt, in dem mittels Methoden der Aktionsforschung und Realexperimenten Erkenntnisse nicht nur über die kooperative Entwicklung des Gängeviertels ermöglicht werden, sondern darüber hinaus auch Erkenntnisse für diese Kooperation, die ihre Umsetzung direkt unterstützen. Um am Ende des Buches 14 Handlungsempfehlungen an Praktiker_innen der Kooperation entwickeln zu können, führt Ziehl zunächst in seinen theoretischen Rahmen ein, den die Resilienzforschung bildet, stellt anschließend die für ihn zentrale Methode des Reallabors und die durchgeführten Realexperimente vor, um schließlich die Kooperation zur Entwicklung des Gängeviertels mit dem theoretisch und methodisch entwickelten Instrumentarium zu beschreiben. 
Das Gängeviertel ist ein Gebäudeensemble in der Hamburger Innenstadt, letztes Überbleibsel eines historischen Arbeiter_innenviertels mit engen Gassen. Umgeben ist die historische Bausubstanz heute von hochpreisigen Bürogebäuden. Im Jahr 2008 verkaufte die Stadt Hamburg das Gängeviertel in einem Höchstbieterverfahren an den niederländischen Investor Hanzevast. Trotz Denkmalschutz genehmigte sie den geplanten Abriss und den Neubau von Wohnungen, Büros und Gewerbeflächen. Gegen diese Pläne regte sich schnell Widerstand: Im Sommer 2009besetzten Aktivist_innen das Viertel unter dem Motto „Komm in die Gänge“, darunter Kunst- und Kulturschaffende, die Teile der Gebäude nutzten. Durch ihr unorthodoxes Auftreten, eine breite Mobilisierung zu Kulturveranstaltungen vor Ort und das Anknüpfen an das stadtpolitische Ziel der Förderung der kreativen Klasse (vgl. Twickel 2010; Novy/Colomb 2013) gelang es den Aktivist_innen, den Senat der Stadt Hamburg zu Verhandlungen zu zwingen. Gleichzeitig, so stellte sich heraus, war der Investor durch die Finanzkrise seit 2007 angeschlagen. So konnte der Verkauf rückabgewickelt werden und das Gängeviertel blieb in öffentlicher Hand. Die Aktivist_innen konnten aus dieser Position als Retter_innen des Gängeviertels die legitime Forderung nach einer außergewöhnlichen Beteiligung an der weiteren Entwicklung des Viertels stellen. Damit begann ein Kooperationsverfahren zwischen den Aktiven des Gängeviertels auf der einen Seite, organisiert in einem Verein und später einer Genossenschaft, und verschiedenen staatlichen Stellen der Stadt Hamburg und des Bezirks Mitte auf der anderen Seite, das bis heute andauert.

Eine solche kooperative Stadtentwicklung trägt laut Ziehl zentral zu einer zukunftsfähigen Stadtentwicklung bei, ein Konzept, das er mithilfe der urbanen Resilienzforschung entwickelt. Diese beschäftigt sich damit, wie es gelingen kann, Städte an äußere und innere Krisen anzupassen. Ziehl betont, dass „eine reine Anpassung an Krisen und ihre Folgen nicht ausreicht, sondern auch grundlegende Transformationen urbaner Teilsysteme notwendig sind [...], [weil] die Art und Weise, wie Städte heute entwickelt werden, selbst maßgeblich zu Störungen und Krisen beitragen kann" (Ziehl 2020: 9). Unter scheinbar absichtlicher Umgehung des Kapitalismusbegriffs skizziert der Autor, dass es einer sozial-ökologischen Transformation und einer Abkehr vom Paradigma der wachstumsorientierten Stadtentwicklung bedarf, um eine zukunftsfähige Stadtentwicklung zu ermöglichen. Ein Baustein dessen sei die intensivierte Kooperation zwischen Zivilgesellschaft und staatlichen Akteuren. Ziehl argumentiert, dass Bürger_innenorganisationen zur Resilienz von Städten beitragen. Dabei überträgt er die anhand von sozio-technischen Infrastrukturen entwickelten Indikatoren für Resilienz (Redundanz und Modularität, Diversität, Vernetzung, Mehrfunktionalität, Innovation, Selbstorganisation) auf den Kooperationsprozess im Gängeviertel beziehungsweise auf die Forderungen der Aktivist_innen zur Entwicklung des Gängeviertels. Diese Übertragung überzeugt für das gewählte Beispiel, wenn sie auch manchmal etwas gekünstelt wirkt: Die von den Aktivist_innen vorgeschlagene kleinteilige Nutzung in Selbstverwaltung entspricht den Indikatoren für Resilienz. Die angedeutete Generalisierung, Bürger_innenorganisationen würden immer für resilientere und damit nachhaltigere Städte sorgen, steht dagegen auf wackligen Füßen. Eine normative Sicht auf zivilgesellschaftliche Gruppen scheint hier auszuklammern, dass deren 
Forderungen keineswegs immer weitsichtig sind oder auf soziale oder ökologische Gerechtigkeit zielen.

Umso stärker überzeugt Ziehls Analyserahmen für die Kooperation zwischen Aktivist_innen und Verwaltung. Deren Effekt systematisiert er auf sechs Ebenen: Auf der subjektiven Ebene setzt durch die Kooperation ein Bewusstseinswandel ein; auf der Verfahrensebene werden neue Wege der Zusammenarbeit erprobt; auf der strukturellen Ebene passen sich Organisationen an die Erfordernisse von Kooperationen an; auf der Gesetzesebene werden inhaltliche Änderungen wirksam; auf der politischen Ebene soll die Rollevon Bürger_innen gestärkt werden und auf der diskursiven Ebene gelingt schließlich eine Beeinflussung des öffentlichen Diskurses. Zur Untersuchung seines Fallbeispiels stellt Ziehl das Governance-Netzwerk der Kooperation dar und unterteilt den Kooperationsprozess in einzelne Phasen. Durch Ersteres gelingt es, die Komplexität der Akteurskonstellation und deren widerstreitende Interessen - auch jeweils innerhalb der zivilgesellschaftlichen Seite und der Verwaltung/Politik - deutlich werden zu lassen, durch Letzteres wird der mittlerweile über zehnjährige Aushandlungsprozess fassbar.

Die erste Kooperationsphase umfasst die Zeit vom Rückkauf des Gebäudekomplexes 2009 bis zum Abschluss einer Kooperationsvereinbarung im Jahr 2011. In dieser Zeit wird ein Integriertes Entwicklungskonzept für das Gängeviertel entwickelt, in dem einzelne Forderungen der Aktivist_innen wie die Schaffung bezahlbarer Ateliers und Wohnungen berücksichtigt werden, während die Formulierung zur ebenfalls geforderten Selbstverwaltung und langfristigen Nutzungsperspektive der Aktiven schwammig bleibt. Die Aktiven befürchten eine Aufwertung des Gängeviertels nach Abschluss der Sanierung, was sie selbst zu Opfern von Verdrängung machen würde. Sie sehen außerdem kritisch, dass mit der „steg“ ein privatwirtschaftliches Unternehmen als Sanierungsträgerin und als treuhändische Eigentümerin eingesetzt wird. Die Kritik an beiden Punkten führt aufseiten der Aktiven zur Gründung einer Genossenschaft, um als vertraglicher Partner ansprechbar zu sein. Als solcher von der Stadt anerkannt wird die Genossenschaft allerdings erst durch öffentlichen Druck. Die Auseinandersetzung um die Selbstverwaltung mündet in der Unterzeichnung einer Kooperationsvereinbarung, die drei Modelle der Selbstverwaltung vorsieht: Generalmiete, Erbbaurecht oder Kauf durch die Genossenschaft.

Die zweite Phase umfasst die Zeit zwischen der Unterzeichnung der Kooperationsvereinbarung 2011 und dem Planungsstopp 2015. In dieser Phase beginnt die Sanierung einzelner Gebäude im ausgerufenen Sanierungsgebiet. Zum Konfliktkomplex rund um das Thema Selbstverwaltung kommt damit ein weiterer Konflikt: der um geeignete Sanierungsmaßnahmen. Dabei geht es um die Notwendigkeit bestimmter Baumaßnahmen, um Baukosten, Fördervorschriften für energetische Sanierung, um das Verbot, Sanierungen in Eigenleistung zu erbringen, und nicht zuletzt auch um ästhetische Fragen. Die Aktivist_innen sind besorgt, dass ihre Insel des Widerstands im Meer der Bürokomplexe nicht mehr als solche zu erkennen sein könnte, wenn sie mit 08/15-Maßnahmen vereinheitlicht und damit ihres individuellen und informellen Charakters beraubt würde. Der von der Stadt äußerst knapp bemessene Finanz- und Zeitplan tut sein Übriges, den Konflikt anzuheizen, der schließlich in einem Planungsstopp gipfelt. 
Die dritte Kooperationsphase umfasst die Zeit vom Planungsstopp 2015 bis zur Unterzeichnung eines Erbbaurechtsvertrags 2019. In verschiedenen Arbeitsgruppen handeln die Kooperationspartner ihre Konflikte aus und passen darüber hinaus die Strukturen der Kooperation an, indem ein Koordinierungskreis eingerichtet wird, in den Personen mit Entscheidungskompetenz aus ihren jeweiligen Institutionen entsandt werden. Aufseiten der Politik werden nun zuvor als juristisch oder aus Sachzwang als unmöglich abgetane Forderungen plötzlich möglich: Die Genossenschaft als zukünftige Eigentümerin wird als Trägerin von Belegungsbindungen anerkannt und die Höhe des Erbbauzinses entscheidend abgesenkt. Mit der Unterzeichnung des Erbbaurechtsvertrags steht damit der selbstverwalteten Realisierung eines Quartiers mit bezahlbaren Sozialwohnungen, viel Raum für Kunst und Kultur und kleinteiliges Handwerk nichts mehr im Wege. Das Ergebnis des langwierigen Aushandlungsprozesses macht deutlich, dass in dieser kooperativen Entwicklung - im Gegensatz zu einer die Zivilgesellschaft nur beteiligenden - der Einfluss der Aktivist_innen deutlich höher ist.

Diesen Kooperationsprozess begleitete Michael Ziehl von Anfang an als Aktivist und ab 2015 auch als Forscher. Diese Doppelrolle ermöglichte ihm einen tiefen Einblick in die Akteurslandschaft und ihre Zusammenarbeit, was einer_m Außenstehenden wohl nicht gelungen wäre. Gleichzeitig erklärt sie seinen aktivistischen Forschungsansatz, der mit Realexperimenten in den Kooperationsprozess interveniert. Die Methode des Reallabors und seine konkreten Realexperimente stellt Ziehl in einem eigenen Kapitel ausführlich und sehr anschaulich dar:

„Ihr methodisches Prinzip besteht im Wesentlichen darin, dass Forschende in Zusammenarbeit mit Akteur_innen von Transformationsprozessen Experimente durchführen und die daraus gewonnenen Erkenntnisse in ebendiesen Prozess einspeisen, um sie zu erforschen und gleichzeitig zu beeinflussen." (Ziehl 2020: 58 f.)

Ziehl überträgt die in der häufig naturwissenschaftlichen Reallabor-Literatur üblicherweise angeführten drei Wissensformen gewinnbringend auf den Kontext der Stadtforschung. Als Systemwissen fasst er das Wissen über die Entwicklung eines Stadtraums inklusive der dort relevanten Akteure, politischen und rechtlichen Rahmenbedingungen, Machtverhältnisse sowie die Gestaltung der gebauten Umwelt. Ziel- oder Orientierungswissen ist Wissen darüber, welche Entwicklung ein Stadtraum nehmen soll und welche großen und kleinen Änderungen dafür nötig sind. Transformationswissen schließlich ist Wissen über die schrittweise Umsetzbarkeit dieser Änderungen, inklusive des Wissens um die Handlungsrationalitäten, Grundannahmen und Wertvorstellungen der dabei beteiligten Akteure. In Reallaboren soll Wissen der beteiligten Praxisakteure auf allen drei Ebenen generiert werden, der_die Wissenschaftler_in unterstützt dabei - sei es durch die Einführung der Unterscheidung der drei Wissensarten, durch das Einbringen des eigenen fachlichen Wissens oder durch die Anleitung der Praxisakteure zu Reflexion und Wissensvermehrung.

Mit der Zielstellung, Konflikte in der Kooperation zum Gängeviertel aufzuarbeiten und zu überwinden, intervenierte Michael Ziehl mit zwei konkreten Realexperimenten in den Prozess (neben seiner Anwesenheit 
und Ratgeberschaft bei zahlreichen Treffen): In einem Bausymposium 2016 baute er gemeinsam mit den Aktiven des Gängeviertels, Vertreter_innen aus der Verwaltung und Politik und geladenen Expert_innen das System- und Ziel-/Orientierungswissen der Beteiligten über den Stand der Sanierung und mögliche weitere bauliche Schritte aus. Ziel war es, neben der Wissensproduktion auch Empathie zwischen den Kooperationspartner_innen herzustellen. Im Ergebnis wurde den Beteiligten klar, dass sie jeweils unterschiedliche Ziele mit der Entwicklung des Gängeviertels verbanden und die Konflikte teils darauf fußten, aber auch, dass Aspekte des sozialen Miteinanders wie Vertrauen, Wertschätzung und Kompromissbereitschaft bisher nicht genug Beachtung gefunden hatten. Als Folge des Bausymposiums wurde das Kooperationsverfahren angepasst, indem eine professionelle Moderation der Gespräche eingeführt wurde und diese an neutralem Ort stattfanden.

In seinem zweiten Realexperiment, dem Laborbericht, stellte Michael Ziehl seine Erkenntnisse aus der Beobachtung der Kooperation den Praxisakteuren in Form von Handlungsanregungen zur Verfügung - ein Angebot, das allerdings zunächst nur die Aktiven des Gängeviertels annahmen. Politik und Verwaltung schlugen die Teilnahme an der Präsentation des Berichts aus. Da er in schriftlicher Form vorliegt, ist von einer späteren, zumindest teilweise vollzogenen Rezeption auszugehen, denn eine der Empfehlungen zur Anpassung des Verfahrens wurde mit der Einführung des Koordinierungskreises umgesetzt.

Ziehls Anliegen war es nicht nur, Handlungsempfehlungen für den konkreten Kooperationsprozess des Gängeviertels zu generieren, sondern genereller für alle Kooperationsprozesse zwischen zivilgesellschaftlichen Gruppen und städtischen Verwaltungen, auch wenn er auf die notwendige Beschränktheit der Erkenntnisse anhand eines Fallbeispiels hinweist. Folgerichtig schließt sein Buch mit 14 Handlungsempfehlungen für die an Kooperationen beteiligten zivilgesellschaftlichen und staatlichen Akteure, die Ziehl aus seiner Analyse herleitet und erläutert. Elf dieser Forderungen beziehen sich auf die Akteure, ihre Beziehungen und die Organisation ihrer Kooperation:

- Vertrauen zwischen den Akteuren herstellen und bewahren

- führungsstarke Vertreter_innen mandatieren

- Entscheidungs- und Handlungsmacht ausgewogen verteilen

- übergeordnete Ziele definieren

- übergeordnete Koordinationsgremien schaffen

- Organisationsstrukturen anpassen

- mit Zeit und Geld Konflikten entgegenwirken

- ehrenamtlich Engagierte finanziell unterstützen

- Privilegien demokratisch legitimieren

- Netzwerke nutzen und Öffentlichkeit einbeziehen

- Intermediäre und Expert_innen einbeziehen

Zwei Empfehlungen sind eher inhaltlicher Natur:

- Besitzrechte langfristig regeln

- Vergabe von öffentlichen Grundstücken/Gebäuden an Nutzung orientieren, nicht an Verkehrswert 
Eine Empfehlung weist schließlich über den einzelnen Kooperationsprozess hinaus:

- Reallabore initiieren und Kooperationsmodelle entwickeln

Diese Handlungsempfehlungen beziehen sich teils auf die inhaltliche Ebene der Kooperation, teils auf die subjektive Ebene der Beteiligten und teils auf diejenige des Verfahrens. Es deuten sich zwei Konflikte an, die Ziehl erwähnt, aber nicht aufzulösen vermag: Erstens haben sich die Aktiven des Gängeviertels infolge der finanziellen Entschädigung von Engagierten und der Anpassung von Organisationsstrukturen an die Anforderungen einer zeitaufwendigen Kooperation professionalisiert. Dies führte im Gängeviertel zu Konflikten und dem (zeitweisen) Rückzug vieler Aktivist_innen, da dadurch Wissenshierarchien und formalisierte Abläufe entstehen, die mit ehrenamtlichem Engagement nicht zu meistern sind. Wie dieser Widerspruch zwar nicht aufgelöst, aber doch bearbeitet werden könnte, deutet Ziehl nur an, nämlich indem möglichst viel Transparenz innerhalb und außerhalb der zivilgesellschaftlichen Gruppe hergestellt wird. Der zweite Widerspruch, der sich in den Handlungsempfehlungen verbirgt, ist der Anspruch, dass solche Kooperationsprozesse keine Ausnahme bleiben, sondern sich vervielfältigen oder gar zur Regel werden. Gleichzeitig schildert das Buch eindringlich die hohen Anforderungen, die dabei an zivilgesellschaftliche Gruppen gestellt werden. Ob es gelingt, diesen überall und vor allem durch die Beteiligung aller gesellschaftlicher Gruppen gerecht zu werden, bleibt fraglich.

Diese beiden offenen Enden des Buches tun seiner Qualität allerdings keinen Abbruch, im Gegenteil: Sie regen zum Weiterdenken an. Michael Ziehl ist ein gewichtiger Beitrag zur kritischen Stadtforschung gelungen, in dem er einen Kooperationsprozess seziert, dafür geeignete Analyserahmen anbietet und Wesentliches zur Übertragung der Methode Reallabor auf den städtischen Kontext leistet (vgl. Räuchle/Schmiz 2020). Anhand von Beispielen wie der Entwicklung des Gängeviertels und mit Studien wie der von Michael Ziehl lässt sich auch die Debatte um partizipative Stadtplanung weiterführen: Führt eine Beteiligung immer zur Einhegung zivilgesellschaftlicher Akteure und Forderungen? Unter welchen Umständen und mit welchen Praktiken ist diese Einhegung zu umgehen? Dem Buch ist eine breite Leser_innenschaft zu wünschen, nicht nur in der Stadtforschung, sondern auch in der stadtpolitischen Szene und in den Verwaltungsstuben unserer Städte.

Die Bauhaus-Universität Weimar unterstützt die Publikation dieses Beitrags durch eine institutionelle Vereinbarung zur Finanzierung von Publikationsgebühren.

\section{Autor_innen}

Lisa Vollmer ist interdisziplinäre Stadt- und Bewegungsforscherin. Ihre Forschungsinteressen sind soziale Bewegungen, Wohnungspolitik und politische Theorie.

lisa.vollmer@uni-weimar.de 


\section{Literatur}

Novy, Johannes / Colomb, Claire (2013): Struggling for the right to the (creative) city in Berlin and Hamburg. New urban social movements, new „spaces of hope”? In: International Journal of Urban and Regional Research 37/5, 1816-1838.

Räuchle, Charlotte / Schmiz, Antonie (2020): „Wissen Macht Stadt: Wie in Reallaboren Stadt verhandelt und Wissen produziert wird“. In: sub\urban. zeitschrift für kritische stadtforschung 8/3, 31-52.

Twickel, Christoph (2010): Gentrifidingsbums. Oder eine Stadt für alle. Hamburg: Ed. Nautilus.

Ziehl, Michael (2020): Koproduktion urbaner Resilienz. Das Gängeviertel in Hamburg als Reallabor für eine zukunftsfähige Stadtentwicklung mittels Kooperation von Zivilgesellschaft, Politik und Verwaltung. Hamburg: jovis. 